\title{
Impaired whole-body heat loss in type 1 diabetes during exercise in the heat: a cause for concern?
}

\author{
Sean R. Notley ${ }^{1}$ - Martin P. Poirier ${ }^{1} \cdot$ Jane E. Yardley ${ }^{2,3} \cdot$ Ronald J. Sigal ${ }^{1,4,5,6,7} \cdot$ Glen P. Kenny ${ }^{1,7}$
}

Received: 30 January 2019 / Accepted: 11 March 2019 / Published online: 2 April 2019

(C) Springer-Verlag GmbH Germany, part of Springer Nature 2019

Keywords Body core temperature $\cdot$ Calorimetry $\cdot$ Diabetes $\cdot$ Exercise $\cdot$ Heat stress $\cdot$ Thermoregulation

To the Editor: Although regular exercise is recommended for type 1 diabetes management [1], exercise in hot conditions may pose a health concern [2]. This is primarily because even patients without neuropathy display impaired cutaneous vasodilation [3] and sweating [4], especially during vigorous exercise [5], which may increase dry heat gain by reducing bloodborne heat delivery to the skin and attenuate evaporative heat loss. The resulting reductions in total heat loss (dry + evaporative heat loss) can elevate heat illness risk by exacerbating body heat storage and the subsequent increase in body core temperature [6]. However, since those previous studies [3-5] measured cutaneous vasodilation and sweating at only a handful of small surfaces $\left(\sim 1-3 \mathrm{~cm}^{2}\right)$ on the body, it remains unclear whether such impairments translate into clinically meaningful decrements in whole-body total heat loss (i.e. from all body surfaces). We therefore used our unique direct air calorimeter (the gold standard method for measuring whole-body heat exchange) [7] to examine the effects of type 1 diabetes on whole-body total heat loss and body heat storage for the first time during light, moderate and vigorous exercise in the heat.

Glen P. Kenny

gkenny@uottawa.ca

1 Human and Environmental Physiology Research Unit, School of Human Kinetics, 125 University, Room 367, Montpetit Hall, University of Ottawa, Ottawa, ON K1N 6N5, Canada

2 Augustana Faculty, University of Alberta, Camrose, AB, Canada

3 Alberta Diabetes Institute, Edmonton, AB, Canada

4 Department of Medicine, University of Calgary, Calgary, AB, Canada

5 Department of Community Health Sciences, University of Calgary, Calgary, AB, Canada

6 Department of Cardiac Sciences, University of Calgary, Calgary, AB, Canada

7 Clinical Epidemiology Program, Ottawa Hospital Research Institute, Ottawa, ON, Canada
Following written informed consent, 28 habitually active young adults (aged 18-37 years) with (ten men, four women) and without (ten men, four women; control) type 1 diabetes, but of similar age, aerobic fitness and body morphology, participated in an experimental trial approved by the University of Ottawa Research Ethics Board. Participants with type 1 diabetes had been diagnosed $\geq 5$ years earlier, had an $\mathrm{HbA}_{1 \mathrm{c}}$ of $6.2-9.2 \%$ (44-77 mmol$/ \mathrm{mol})$, and no diagnosed diabetesrelated complications. Participants were instructed to arrive well hydrated, having followed their normal insulin therapy (type 1 diabetes: pump, $n=8$; injections, $n=6$ ), having eaten their usual breakfast and having abstained from exercise, alcohol, caffeine and anti-inflammatory drugs for $>24 \mathrm{~h}$.

After confirming euhydration (urine specific gravity: $<1.025)$, participants entered the direct calorimeter $\left(35^{\circ} \mathrm{C}\right.$, relative humidity $\sim 20 \%$; humidex $36^{\circ} \mathrm{C}$ ) wearing shorts, sleeveless top (women) and sandals. Participants completed $30 \mathrm{~min}$ of seated rest and three $30 \mathrm{~min}$ bouts of semirecumbent cycling at metabolic heat production rates of 200 (light), 250 (moderate), and $300 \mathrm{~W} / \mathrm{m}^{2}$ (vigorous), each followed by $30 \mathrm{~min}$ recovery. These work rates represented $\sim 37 \%, 47 \%$ and $56 \%$ of peak aerobic power, respectively, and ensured the heat load was matched between groups. Indirect calorimetry (Moxus system, AEI Technologies, Bastrop, TX, USA) was used to derive metabolic heat production (metabolic rate - external work). The direct calorimeter measured whole-body dry and evaporative heat exchange [7]. Body core temperature was measured via the oesophagus ( $n=9$ per group) or rectum ( $n=5$ per group) using a Mon-a-therm Temperature Probe (Mallinckrodt Medical, St Louis, MO, USA). Blood samples were drawn from an indwelling catheter during the final $5 \mathrm{~min}$ of rest and each exercise period from the type 1 diabetes $(n=14)$ and control $(n=12)$ participants, and analysed for blood glucose concentrations $(\mathrm{mmol} / \mathrm{l})$ by an external laboratory (Dynacare, Brampton, ON, Canada).

Data are reported as means over the final $5 \mathrm{~min}$ of rest and each exercise period. Body heat storage $(\mathrm{kJ})$ during each 
exercise period represents the temporal summation of metabolic heat production and total heat loss (dry + evaporative heat loss). Body core temperature changes from rest were normalised to $37^{\circ} \mathrm{C}$ to account for baseline variation. Data were analysed using a two-way, mixed-model ANOVA (using Prism 8, GraphPad, La Jolla, CA, USA) with factors of group (type 1 diabetes, control) and time (rest, light, moderate, vigorous). Post hoc between-group comparisons were carried out using unpaired two-tailed $t$ tests $(\alpha=0.05$ for all comparisons). Based on previously reported type 1 diabetesrelated reductions in sweating [5], $\geq 13$ participants per group were required to detect between-group differences of similar effect size with $80 \%$ statistical power. Our analysis $(n=14$ per group) was therefore adequately powered.

Metabolic heat production (Fig. 1a) and dry heat loss (Fig. 1b) did not differ between groups. However, evaporative heat loss was attenuated in participants with type 1 diabetes relative to control participants during vigorous exercise $(p=0.03)$
Fig. 1 Metabolic heat production (a), and dry (b), evaporative (c) and total heat loss (d), body heat storage (e), body core temperature (f), blood glucose (g) and the change in blood glucose (h) during 30 min of rest and light, moderate and vigorous exercise in dry heat $\left(35^{\circ} \mathrm{C}, 20 \%\right.$ relative humidity). Data are means (SD) of the final 5 min of rest and exercise in participants with (ten men, four women; T1D) and without (ten men, four women; control) type 1 diabetes. Negative values for dry heat loss denote heat gain from the environment. $*$ Significantly different $(p<0.05)$ vs control
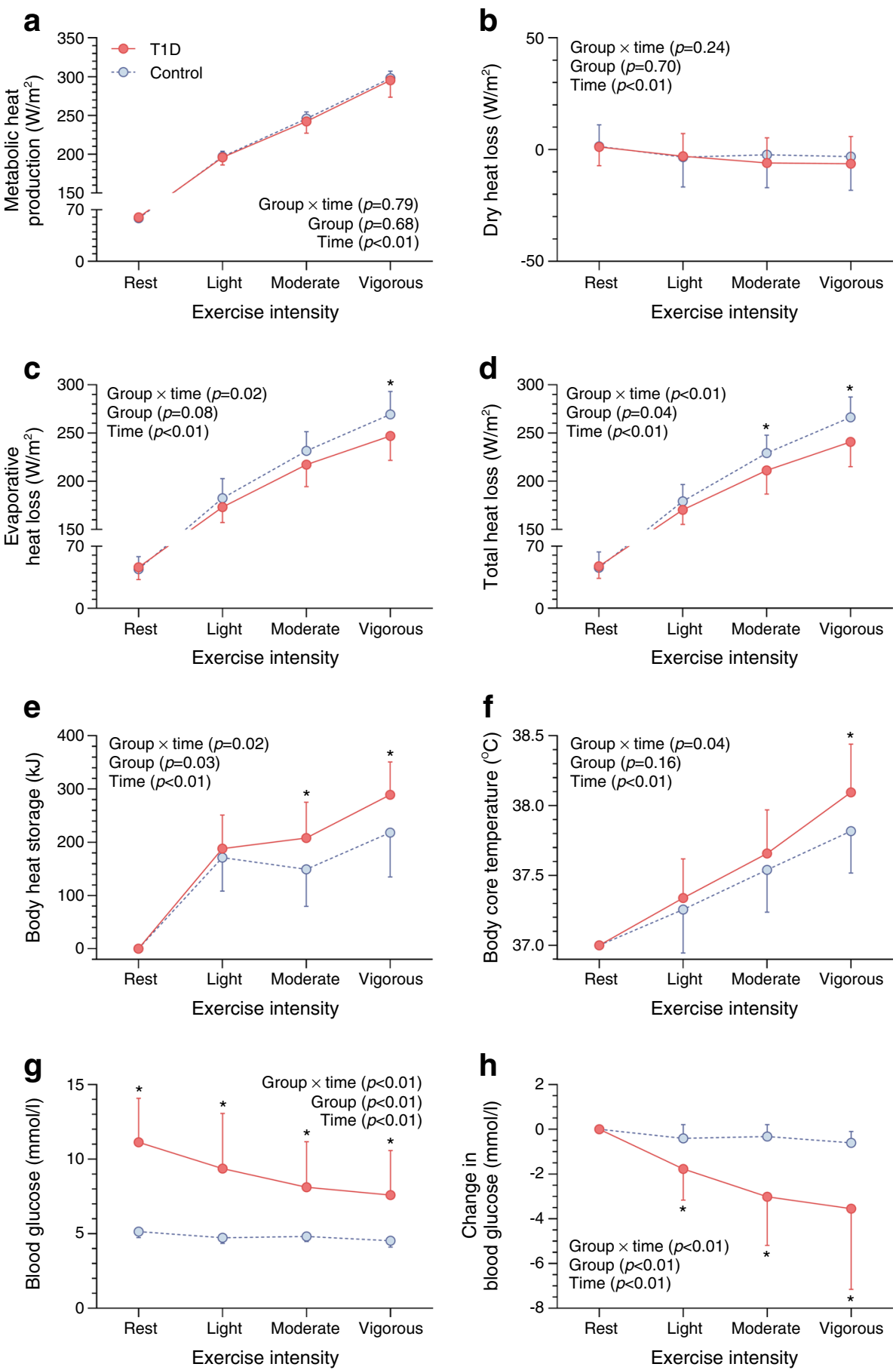
(Fig. 1c). Total heat loss was also lower in participants with type 1 diabetes during moderate $(p=0.04)$ and vigorous exercise ( $p=0.01$ ) (Fig. 1d). Consequently, participants with type 1 diabetes displayed higher body heat storage during moderate $(p=0.03)$ and vigorous exercise $(p=0.02)$ (Fig. 1e) and a higher body core temperature during vigorous exercise relative to control $(p=0.03)$ (Fig. 1f). Blood glucose was higher in type 1 diabetes relative to control throughout (all $p<0.01$ ) (Fig. 1g), albeit a greater reduction from rest occurred in type 1 diabetes during exercise (all $p<0.05$ ) (Fig. 1h).

Observations from this experiment indicate that relative to their matched controls, young patients with type 1 diabetes without clinically evident neuropathy display similar wholebody dry heat exchange, but impaired whole-body evaporative heat loss during exercise, perhaps due to attenuated sweat gland innervation [8]. This impairment worsened with increasing exercise intensity, resulting in a mean reduction in total heat loss of $\sim 10 \%$ and a subsequent mean increase in body heat storage of $\sim 35 \%$ in type 1 diabetes during moderate and vigorous exercise. Importantly, this increase in body heat storage represented a between-group difference in body temperature change equivalent to $\sim 0.5^{\circ} \mathrm{C} / \mathrm{h}$ (assuming a body mass of $70 \mathrm{~kg}$ with a specific heat capacity of $3.47 \mathrm{~kJ} \mathrm{~kg}^{-1}$ ${ }^{\circ} \mathrm{C}^{-1}$ ), which could elevate heat illness risk and result in heat stroke during exercise of longer duration and/or in hotter conditions [6]. Furthermore, while blood glucose was elevated throughout exercise in type 1 diabetes, one might expect greater reductions in heat loss and even higher body heat storage in individuals with less well-controlled diabetes and/ or diabetic neuropathy [2].

Together, our findings provide two key outcomes that advance understanding of thermoregulation in type 1 diabetes. First, although type 1 diabetes has been shown to attenuate cutaneous blood flow [3], those reductions do not appear to modify whole-body dry heat exchange, irrespective of the exercise-induced heat load. Second, a lowered rate of sweat secretion in type 1 diabetes $[4,5]$ can cause reductions in whole-body evaporative heat loss that result in clinically meaningful increases in body heat storage, albeit only during moderate-to-vigorous exercise. While there is a need for larger confirmatory studies, ideally over a broader range of environmental conditions (e.g. hot humid conditions, higher air flow), we suggest that exercise in the heat may pose a health concern that requires consideration in clinical guidelines for exercise management in type 1 diabetes.

Acknowledgements We thank S. Dervis, formerly from the Human and Environmental Physiology Research Unit (www.HEPRU.ca), who performed data collection.
Data availability Data are available on request from the corresponding author.

Funding This project was supported by the Canadian Institutes of Health Research (grant no. 286363; funds held by GPK and RJS). SRN is supported by a Postdoctoral Fellowship from HEPRU. MPP was supported by an NSERC Canada Alexander Graham Bell Graduate Scholarship (CGS-D). GPK is supported by a University of Ottawa Research Chair.

Duality of interest The authors declare that there is no duality of interest associated with this manuscript.

Contribution statement SRN and GPK conceptualised and designed the research; MPP performed data collection. SRN performed statistical analysis, prepared figures and drafted manuscript; SRN, GPK, JEY and RJS interpreted results; all authors edited, revised and approved the final version. GPK is the guarantor of this work and, as such, had full access to all the data and takes responsibility for the integrity of the data and the accuracy of the data analysis.

\section{References}

1. American Diabetes Association (2019) 5. Lifestyle management: standards of medical care in diabetes-2019. Diabetes Care 42(Suppl 1):S46-S60. https://doi.org/10.2337/dc19-S005

2. Yardley JE, Stapleton JM, Carter MR, Sigal RJ, Kenny GP (2013) Is whole-body thermoregulatory function impaired in type 1 diabetes mellitus? Curr Diabetes Rev 9(2):126-136

3. Wilson SB, Jennings PE, Belch JJ (1992) Detection of microvascular impairment in type I diabetics by laser Doppler flowmetry. Clin Physiol 12(2):195-208. https://doi.org/10.1111/j.1475-097X.1992. tb00306.x

4. Hoeldtke RD, Bryner KD, Hoeldtke ME et al (2006) Sympathetic sudomotor disturbance in early type 1 diabetes mellitus is linked to lipid peroxidation. Metabolism 55(11):1524-1531. https://doi.org/ 10.1016/j.metabol.2006.06.023

5. Carter MR, McGinn R, Barrera-Ramirez J, Sigal RJ, Kenny GP (2014) Impairments in local heat loss in type 1 diabetes during exercise in the heat. Med Sci Sports Exerc 46(12):2224-2233. https:// doi.org/10.1249/MSS.0000000000000350

6. Kenny GP, Wilson TE, Flouris AD, Fujii N (2018) Heat exhaustion. Handb Clin Neurol 157:505-529

7. Kenny GP, Notley SR, Gagnon D (2017) Direct calorimetry: a brief historical review of its use in the study of human metabolism and thermoregulation. Eur J Appl Physiol 117(9):1765-1785. https://doi. org/10.1007/s00421-017-3670-5

8. Gibbons CH, Illigens BM, Wang N, Freeman R (2009) Quantification of sweat gland innervation: a clinical-pathologic correlation. Neurology 72(17):1479-1486. https://doi.org/10.1212/ WNL.0b013e3181a2e8b8

Publisher's note Springer Nature remains neutral with regard to jurisdictional claims in published maps and institutional affiliations. 